\title{
Taurine induces the apoptosis of breast cancer cells by regulating apoptosis-related proteins of mitochondria
}

\author{
XIALI ZHANG ${ }^{*}$, HONGFEI LU ${ }^{2 *}$, YIBING WANG ${ }^{3}$, CHUNJU LIU $^{4}$, \\ WEIFENG ZHU ${ }^{2}$, SHUANGYAN ZHENG ${ }^{1}$ and FUSHENG WAN ${ }^{1,2}$ \\ ${ }^{1}$ Department of Laboratory Animal Science, ${ }^{2}$ Department of Biochemistry and Molecular Biology, Basic Medical College, \\ ${ }^{3}$ Institute of Urology, the First Affiliated Hospital, Nanchang University; ${ }^{4}$ Clinical Laboratory of \\ Jiangxi University of Traditional Chinese Medicine, Nanchang, Jiangxi 330006, P.R. China
}

Received July 20, 2014; Accepted October 27, 2014

DOI: $10.3892 /$ ijmm.2014.2002

\begin{abstract}
Taurine (Tau), the most abundant free amino acid in humans has numerous potential health benefits through its antioxidant and anti-inflammatory properties. However, limited studies have assessed its effect on tumors and the antitumor mechanism remains unknown. The present study investigated the cellular and molecular changes induced by Tau, leading to the induction of apoptosis in human breast cancer cell lines MCF-7 and MDA-MB-231. MCF-7 is p53

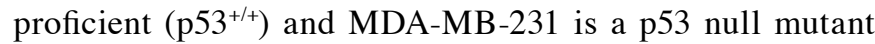
$\left(\mathrm{p} 53^{--}\right)$. Cell proliferation and viability were assessed by MTT. Flow cytometry and hoechst 33342 fluorescent staining were employed to detect apoptosis. Spectrophotometry was used to detect caspase-3 activity. Reverse transcription-polymerase chain reaction and western blot analysis were used to detect the levels of mRNA and proteins of p53-upregulated modulator of apoptosis (PUMA), Bax and Bcl-2. Finally, the affect of Tau on the growth of MDA-MB-231-cell-nude mice xenografts was examined. In the study, Tau inhibited growth and induced apoptosis of the two cell lines in a concentration- and time-dependent manner. Notably, the inhibitory effect of Tau on $\mathrm{p} 53^{-/-}$cancer cells was clearly significant compared to the p53 $3^{+/+}$cancer cells. Further studies showed that Tau promoted apoptosis in human breast cancer cells and inhibited the growth of tumor in nude mice by inducing the expression of PUMA, which further up- and downregulated the expression of Bax and Bcl-2 protein, giving rise to increased activation of caspase-3. Collectively, these results indicate that Tau is a
\end{abstract}

Correspondence to: Professor Fusheng Wan, Department of Laboratory Animal Science, Nanchang University, 71 Yang Ming East Road, Nanchang, Jiangxi, Nanchang 330006, P.R. China

E-mail: 381609018@qq.com

${ }^{*}$ Contributed equally

Key words: taurine, human breast cancer cells, p53-upregulated modulator of apoptosis potent candidate for the chemotherapy of breast cancer through increasing the PUMA expression independent of $p 53$ status.

\section{Introduction}

In recent years, cancer has become the leading cause of mortality, and it is detrimental to health and quality of life. Environmental chemical carcinogens have been estimated to contribute significantly to the causation of a sizable fraction, perhaps a majority, of human cancers, when exposures are correlated to 'life-style' factors, such as diet (1). Therefore, the anticancer substances in the diet have become of current interest in research for cancer prevention.

Tau, chemically identified as 2-aminoethanesulfonic acid, is a sulfur-containing amino acid of a simple chemical structure. Tau accounts for $\sim 0.1 \%$ of total human body weight and exists in a free-state in all the organs. However, the ability of endogenous Tau synthesis is limited. A large number of studies and clinical applications demonstrated that Tau has extensive physiological effects, and has been identified to be the endogenous anti-injury material. Recently, Tau has been used to in the therapy of diseases, including liver and gallbladder disease (2), cardiovascular disease $(3)$, diabetes $(4,5)$ and cataract $(6,7)$. However, studies on its effect on tumors remains limited and the mechanism of its antitumor ability is ambiguous. Tau has been reported to protect cells from oxidant-induced injury by scavenging strong oxidant and cytotoxic agents (8). Therefore, Tau as an effective antioxidant may hinder an increase in reactive oxygen species in tumors, thereby reducing the development of cancer (9). In addition, Tau could have anti-tumor activity by downregulation of MMP-2, upregulation of N-acetylgalactosaminytransferase, and inhibition of the potential invasion and metastasis induced by ionizing radiation (10).

Apoptosis, also known as programmed cell death, is an autonomous cell death based on a genetic program. Bcl-2 family members focus the majority of their efforts on mitochondria, which play a pivotal role in the apoptotic cells. Bcl-2 family members share homology in one to four conserved regions designated the $\mathrm{Bcl}-2$ homology $(\mathrm{BH})$ domains; $\mathrm{BH} 1$, $\mathrm{BH} 2, \mathrm{BH} 3$ and $\mathrm{BH} 4$. Among them, the $\mathrm{BH} 3$ domain plays an important role in apoptosis by mediating the interaction 
among the Bcl-2 family members. Once the cells are exposed to apoptotic stimuli, the $\mathrm{BH} 3$-only protein and other regulators translocate to mitochondria, where they promote the release of pro-apoptotic factors through changing the mitochondrial permeability.

PUMA is a member of the BH3-only subgroup of Bcl-2 family proteins. Once exposed to a variety of apoptosis stimuli, the BH3 domain of PUMA interacted directly with anti-apoptotic Bcl-2 family members, inhibiting their interaction with the pro-apoptotic molecules. Thus, PUMA was indicated as the most potential regulator that could trigger apoptosis $(11,12)$. PUMA is an essential apoptotic mediator of p53-dependent and -independent (13) pathways and reported to have a potential binding site for $\mathrm{p} 53$ in its promoter region. In the p53-dependent pathway, within hours of apoptosis stimuli, p53 was recruited to the p53-responsive element, and furthermore, induced the transcription and translation of PUMA $(14,15)$. PUMA can also be induced by other regulators that are independent of $\mathrm{p} 53$, such as transcription factor E2F1 (16), transforming growth factor- $\beta$ (17) and c-Jun kinase (18). A number of studies have confirmed that PUMA plays important roles in tumors (19), cerebral vascular and myocardial hypoxia reoxygenation injury $(20,21)$, and cell apoptosis in multiple cell lines. Therefore, PUMA may be an efficient target for cancer therapy.

In the present study, Tau was used for treatment of the human breast cancer cells lines, MCF-7 and MDA-MB-231, and it was found that it could induce cell apoptosis and repression of the tumor growth in vivo and in vitro. The results indicated that Tau may be useful in the therapeutic target selection for p53-deficient breast cancer.

\section{Materials and methods}

Reagents and antibodies. Tau was purchased from Sigma-Aldrich (Munich, Germany). RPMI-1640 medium, Dulbecco's modified Eagle's medium (DMEM) and fetal bovine serum (FBS) were purchased from Gibco (Gaithersburg, MD, USA). The cDNA Reverse Transcription kit was purchased from Thermo Scientific (Waltham, MA, USA). Anti-GAPDH polyclonal antibody (TA-08), anti-Bcl-2 monoclonal antibody (sc-783) and anti-Bax monoclonal antibody (sc-526) were purchased from Santa Cruz Biotechnology, Inc. (Santa Cruz, CA, USA). Anti-PUMA polyclonal antibody (\#Q9BXH1-4976) was purchased from Cell Signaling Technology (Beverly, MA, USA). Horseradish peroxidase (HRP)-conjugated goat anti-mouse immunoglobulin G (IgG) (ZDR-5307) and goat anti-rabbit IgG (ZDR-5306) were purchased from Beijing ZSGB-Bio (Beijing, China).

Cell lines and animals. The human breast cancer cell lines, MCF-7 and MDA-MB-231, were purchased from the Cell Bank of Type Culture Collection of Chinese Academy of Sciences (Shanghai, China). MCF-7 cells were cultured in DMEM medium supplemented with 10\% FBS, and MDA-MB-231 cells were cultured in RPMI 1640 medium supplemented with $10 \%$ FBS. All the cell lines were maintained at $37^{\circ} \mathrm{C}$ in a humidified atmosphere of $5 \% \mathrm{CO}_{2}$.

Seven-week old female-specific pathogen-free nude mice (BALB/C) (certificate NO. HNASLKJ20121369) were purchased from Hunan Slack King of Laboratory Animal Co., Ltd., (Changsha, China) and maintained in a laminar flow cabinet (Su net Aetna, Suzhou, China) in the Department of Animal Science, Nanchang University (Nanchang, China), at a constant temperature, constant humidity and sterile environment.

Drug treatment. The breast cancer cells were treated with a concentration gradient of Tau (10-160 mM, dissolved in medium without fetal bovine serum) for different time periods. The cells treated with $4 \mu \mathrm{g} / \mathrm{ml}$ cisplatin were used as a positive control.

MTT assay. Cell viability was determined with the 3-(4, 5-dimethylthiazol-2-yl)-2,5-diphenyltetrazolium bromide (MTT) assay. After treating with Tau for 24, 48 and $72 \mathrm{~h}$, cultures were washed with phosphate-buffered saline (PBS; Solarbio Science and Technology Co., Ltd., Beijing, China). Subsequently, MTT $(0.5 \mathrm{mg} / \mathrm{ml})$ was added to each well and the mixture was incubated at $37^{\circ} \mathrm{C}$ for $4 \mathrm{~h}$. To dissolve formazan crystals, culture medium was replaced with an equal volume of dimethyl sulfoxide. After the mixture was agitated at room temperature for $10 \mathrm{~min}$, the absorbance of each well was determined at $490 \mathrm{~nm}$ using a microplate reader (Bio-Tek, Winooski, VT, USA). The inhibition rate was calculated as follows:

Inhibition rate $(\%)=(1-$ experiment group absorbance/ control group absorbance) $\mathrm{x} 100 \%$

Quantification of apoptosis by flow cytometry. The cells $\left(1-5 \times 10^{5}\right.$ cells) were treated with Tau for the indicated times, washed twice with PBS and resuspended in staining buffer containing $1 \mu \mathrm{g} / \mathrm{ml}$ propidium iodide (PI) and $0.025 \mu \mathrm{g} / \mathrm{ml}$ Annexin V-fluorescein isothiocyanate (FITC). Double-labeling was performed at room temperature for $10 \mathrm{~min}$ in the dark, and cells were immediately analyzed by FACScan and the Cellquest program (Becton Dickinson; Lincoln Park, NJ, USA).

Hoechst33342 staining. Subsequent to adjusting the concentration, the cell suspension was seeded in a 12-well plate, and three parallel holes were designated for each group. On the following day, the cells were treated with $80 \mathrm{mM}$ Tau for $48 \mathrm{~h}$, and $500 \mu \mathrm{l}$ Hoechst 33342 dyes were subsequently added to cover the cells, which were incubated in the dark for 20-30 min. Finally, the unbound dye was removed and the morphology of the apoptotic cells were observed using the fluorescence microscope.

Spectrophotometry analysis. The assay is based on the ability of the active enzyme to cleave a chromophore from the enzyme substrate, Ac-DEVD-pNA (for caspase-3). Cell lysates were prepared and incubated with anti-caspase-3. Immunocomplexes were incubated with peptide substrate in assay buffer (caspase-3 activity assay kit; Beyotime Institute of Biotechnology, Jiangsu, China) for $2 \mathrm{~h}$ at $37^{\circ} \mathrm{C}$. The release of $p$-nitroaniline was monitored at $405 \mathrm{~nm}$. Results are the percentage change in activity compared to the untreated control. 
Table I. Primer sequences for RT-PCR.

\begin{tabular}{llc}
\hline Gene & \multicolumn{1}{c}{ Primer sequence } & Length of product, bp \\
\hline PUMA & Forward: 5'-CCTCAGCCCTCGCTCTCGC-3' & 559 \\
Bcl-2 & Reverse: 5'-CCGATGCTGAGTCCATCAGC-3' & 304 \\
Bax & Forward: 5'-GTGGAGGAGCTCTTCAGGGA-3' & \\
& Reverse: 5'-AGGCACCCAGGGTGATGCAA-3' & 298 \\
GAPDH & Forward: 5'-TTGCTTCAGGGTTTCATCCA-3' \\
GAPDH & Reverse: 5'-CAGCCTTGAGCACCAGTTG-3' & 241 \\
& Forward: 5'-GGCTCTCCAGAACATCAT-3' & 648 \\
\hline
\end{tabular}

RT-PCR, reverse transcription-polymerase chain reaction; bp, base pair.

Reverse transcription-polymerase chain reaction (RT-PCR) analysis. Total RNA was routinely extracted using Trizol (Invitrogen, Carlsbad, CA, USA). RNA concentration and purity were detected using an Eppendorf nucleic acid quantitative determination meter. Subsequently, RNA integrity was observed following gel electrophoresis. GAPDH was used as the internal control. PUMA, Bax and $B c l-2$ levels were detected by RT-PCR. The primers of PUMA, Bax, Bcl-2 and $G A P D H$ are listed in Table I. The PCR amplification products were analyzed in $1.5 \%$ agarose gel electrophoresis. Gel images were captured for strap grayscale analysis. Optical density values of the target genes and the corresponding $G A P D H$ products in each group were detected. The ratio between the target gene and $G A P D H$ was statistically analyzed.

Western blot analysis. After treatment with Tau for $48 \mathrm{~h}$, the protein was collected and denatured, and $30 \mu \mathrm{g}$ protein was separated in sodium dodecyl sulfate-polyacrylamide gels electrophoresis and transferred onto polyvinylidene difluoride membranes by the wet-transfer method. The membranes were incubated with the primary antibodies against PUMA, Bax and Bcl-2 (1:200) overnight at $4^{\circ} \mathrm{C}$, and HRP-labeled second antibodies (1:200) were incubated with membrane for $1.5 \mathrm{~h}$. Finally, the protein signals were detected by the enhanced chemiluminescence detection system. The grayscale of protein bands were scanned using Image-Pro Plus 6.0 (Media Cybernetics, Rockville, MD, USA). The expression relative to GAPDH was analyzed.

In vivo tumor xenograft study. MDA-MB-231 cells $\left(1 \times 10^{6}\right.$ in $200 \mu \mathrm{l}$ ) were injected subcutaneously into the flanks of nude mice, and tumors were allowed to develop until the diameter of the tumor tissue reached a size of $\sim 2-3 \mathrm{~mm}$. The mice were treated with saline or with $200 \mathrm{mg} / \mathrm{kg}$ (i.p total volume $200 \mu \mathrm{l}$ ) Tau every day for 25 days (5 mice/group). The volume of implanted tumors in the dorsal side of the mice was determined every 5 days. All the mice were manipulated in accordance with the Animal Care and Use Guidelines of the China Medical University (Taichung, Taiwan) under a protocol approved by the Institutional Animal Care and Use Committee.
Immunohistochemistry. Immunohistochemical detection of PUMA, Bax and Bcl-2 proteins were performed on 3-mm paraffin sections of formalin-fixed samples according to the manufacturer's instructions. The sections were deparaffinized in xylene three times for $15 \mathrm{~min}$, followed by rehydration in graded alcohol (100\% I, 100\% II, 95\% I, 95\% II, 85\%, 70\% and water) for $2 \mathrm{~min}$. Heat-induced antigen retrieval was performed for $30 \mathrm{~min}$ using the pressure cooker Retriever 2100 with unmasking buffer citrate (Solarbio Science and Technology Co., Ltd.), followed by cooling at room temperature for $30 \mathrm{~min}$ and washing with PBS three times for $5 \mathrm{~min}$. The histostaining was carried out using Histostain-Plus Kits (ZYMED, San Diego, CA, USA) according to the manufacturer's instructions. The antibodies PUMA, Bax and Bcl-2 diluted in 1\% skimmed milk in Tris-buffered saline containing Tween-20 were used. The mean density values were used to indicate the relative expression levels.

Statistical analysis. The results are expressed as the means \pm standard deviation (SD). Data were analyzed by the one-way analysis of variance test by SPSS 17.0 (SPSS, Inc., Chicago, IL, USA) software. $\mathrm{P}<0.05$ was considered to indicate a statistically significant difference.

\section{Results}

Tau kills human breast cancer cells in a time- and dose-dependent manner. To investigate the potential cell growth inhibition by Tau in breast cancer, the effect of Tau on cell proliferation was first examined in MCF-7 and MDA-MB-231 cells. As shown in Fig. 1A and B, Tau significantly inhibited cell growth in the cancer cell lines in a time- and dose-dependent manner. Calculation of the $\mathrm{IC}_{50}$ value for the MCF-7 and MDA-MB-231 cells at 24, 48 and $72 \mathrm{~h}$ were 284.50, 133.61 and 106.40, and 617.99, 127.21 and $90.46 \mathrm{mM}$, respectively. This showed that the inhibitory effect of Tau was more evident on $\mathrm{p} 53^{-/-}$cells compared to $\mathrm{p} 53^{+/+}$cells. After $48 \mathrm{~h}$ of treatment with different concentrations of Tau, microscopic analysis found that the cell density was significantly decreased, there was membrane shrinkage and cell debris was found (Fig. 1C and D). To eliminate Tau toxicity on cell growth, the same 

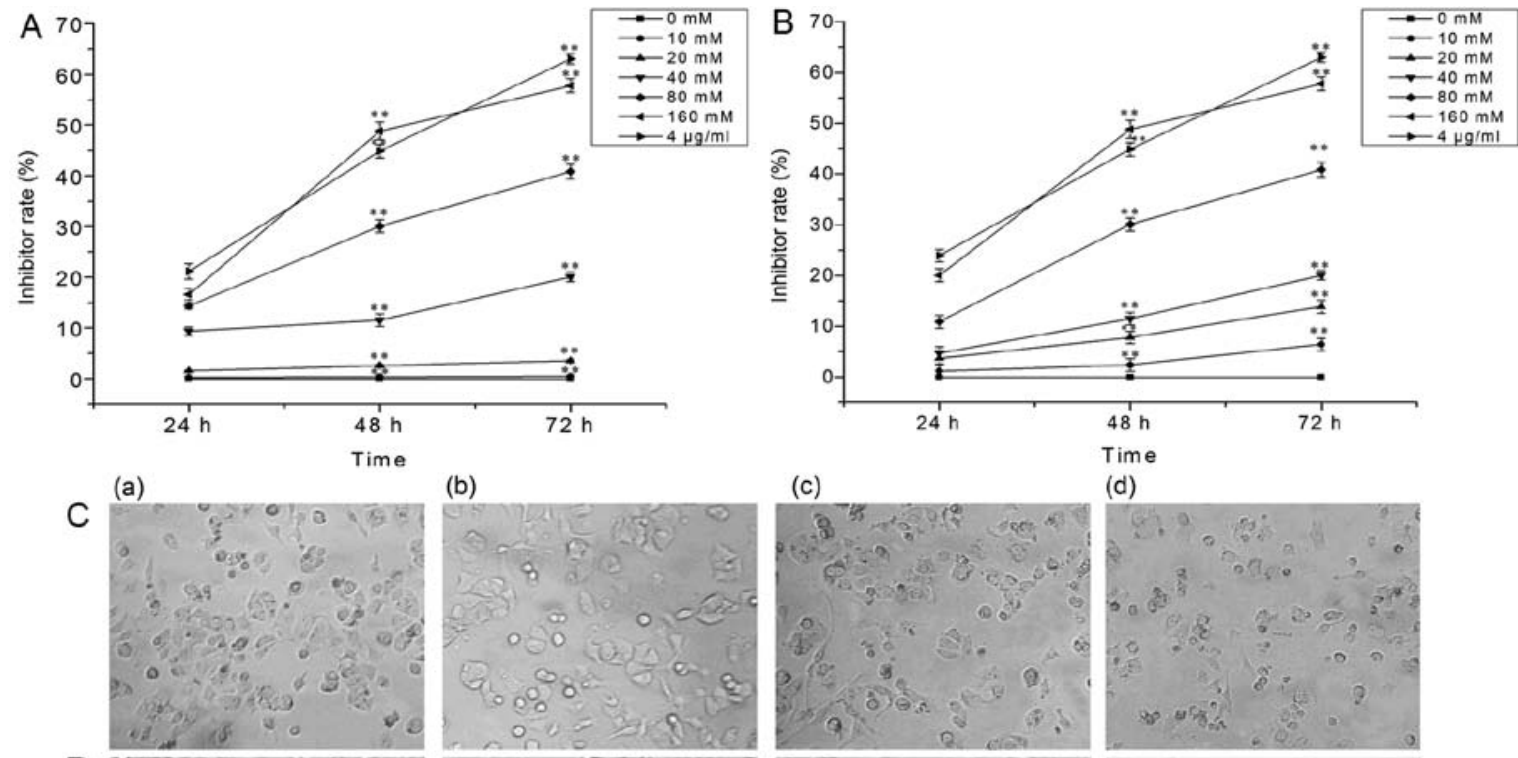

(d)
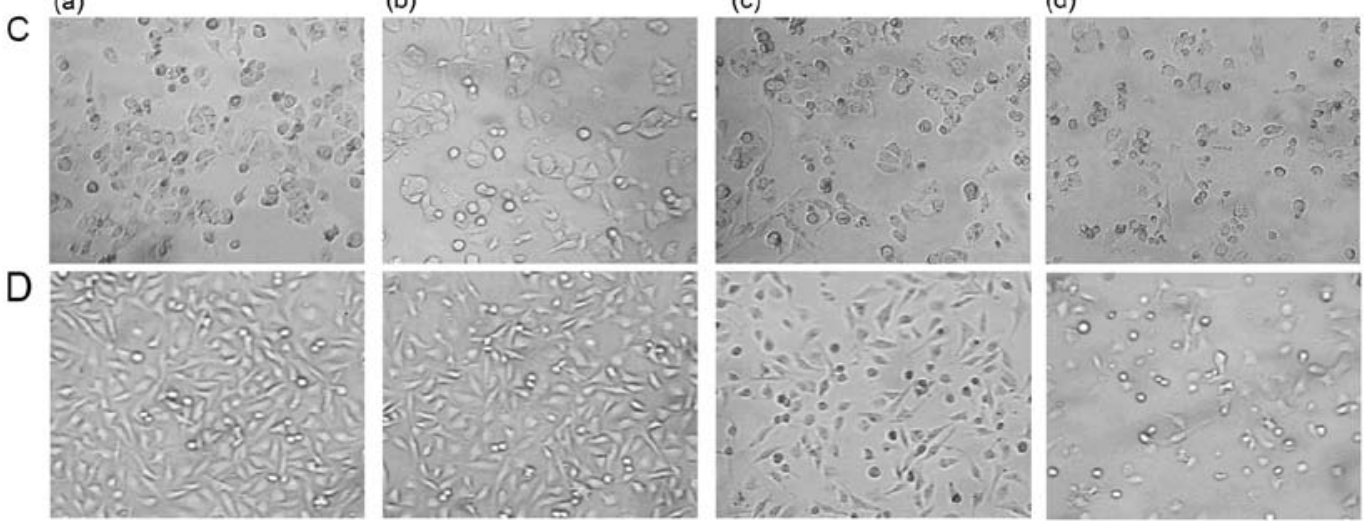

Figure 1. Tau inhibits human breast cancer cell proliferation. (A) MCF-7 and (B) MDA-MB-231 cells were treated with Tau (0, 10, 20, 40, 80 and 160 mM) for the indicated times $(24,48$ and $72 \mathrm{~h})$, followed by assessment of the inhibition rate by the MTT assay $(\mathrm{n}=3)$. Cell morphology under the microscope for (C) MCF-7 and (D) MDA-MB-231 cells (magnification, 200x). (a) 0, (b) 40, (c) 80 and (d) $160 \mathrm{mM}$ Tau. ${ }^{* *} \mathrm{P}<0.01$ compared to the control group.

dose and time were used as the human kidney epithelial cells 293T. Tau, even at the highest dose of $160 \mathrm{mM}$, showed no effect on the cellular viability of $293 \mathrm{~T}$ cells (data not shown).

Tau induces apoptosis in MCF-7 and MDA-MB-231 cells. To evaluate whether the cell death observed in the cells following Tau treatment may occur through apoptosis, Annexin v-FITC/PI and Hoechst 33342 fluorescent staining were utilized to observe the apoptotic rates and morphological changes in the cell lines in response to Tau. The apoptotic rates in the cells lines were significantly increased in a concentration-dependent manner, and the pro-apoptotic effect was more significant in $\mathrm{p} 53^{-1-}$ compared to $\mathrm{p}^{+/+}$cancer cells (Fig. 2A-D). Hoechst 33342 staining showed that untreated cells had round and light-blue nuclei with a high cell density. By contrast, the $80 \mathrm{mM}$ Tau-treated groups exhibited a lower cell density and more apoptotic cells that were densely stained. The typical feature of apoptosis, the apoptotic body, was observed (Fig. 2E).

Spectrophotometry detected caspase- 3 activity in human breast cancer cells treated with Tau (40,80 and $160 \mathrm{mM}$ ) for $48 \mathrm{~h}$. Caspase- 3 activity in the control group was preset to 1 . The results showed that Tau increased caspase- 3 activities dose-dependently in the cells (Fig. 3).

Tau induces the expression of apoptosis-associated genes. To investigate the effect of Tau on PUMA and the associated genes, MCF-7 and MDA-MB-231 were cultured in the absence or presence of 40,80 and $160 \mathrm{mM}$ Tau for $48 \mathrm{~h}$ and the mRNA and protein levels of PUMA, Bax and $\mathrm{Bcl}-2$ were examined in the cell lysates.
The results of the RT-PCR assay showed that the expression of the PUMA gene was significantly upregulated in the cells with increasing concentrations of Tau. For the other apoptosis-related genes, the expression of the pro-apoptotic gene Bax was upregulated, the anti-apoptotic gene $\mathrm{Bcl}-2$ was downregulated and the $\mathrm{Bax} / \mathrm{Bcl}-2$ ratios were increased (Fig. 4).

The western blot assay showed that PUMA and Bax proteins expression were significantly increased and Bcl-2 was significantly decreased in the cells (Fig. 5).

Effect of Tau on the growth of human breast cancer cells in nude mice. On the basis of the Tau-induced apoptotic effect exhibited in vitro, whether Tau possessed antitumor activities in vivo was examined. Therefore, xenografts of MDA-MB-231 cells in nude mice were established; as the diameter of tumor tissue was $\sim 2-3 \mathrm{~mm}$, the mice were divided into two groups and treated with saline or $200 \mathrm{mg} / \mathrm{kg}$ Tau. The tumor volumes were measured every 5 days and a growth curve was generated (Fig. 6A). After 25 days, mice were sacrificed, and the tumor weights were measured (Fig. 6B). Subsequently, the tumor growth inhibition rate induced by Tau was calculated (Fig. 6C).

As depicted by immunohistochemical staining of tumors at the time of sacrificing the mice in the MDA-MB-231 subcutaneous xenograft experiment, PUMA, Bax and Bcl-2 were present in the cytoplasm, and the positive expression of PUMA and Bax protein were upregulated in the Tau group, whereas the positive expression of $\mathrm{Bcl}-2$ protein was downregulated (Figs. 6D and 7). 

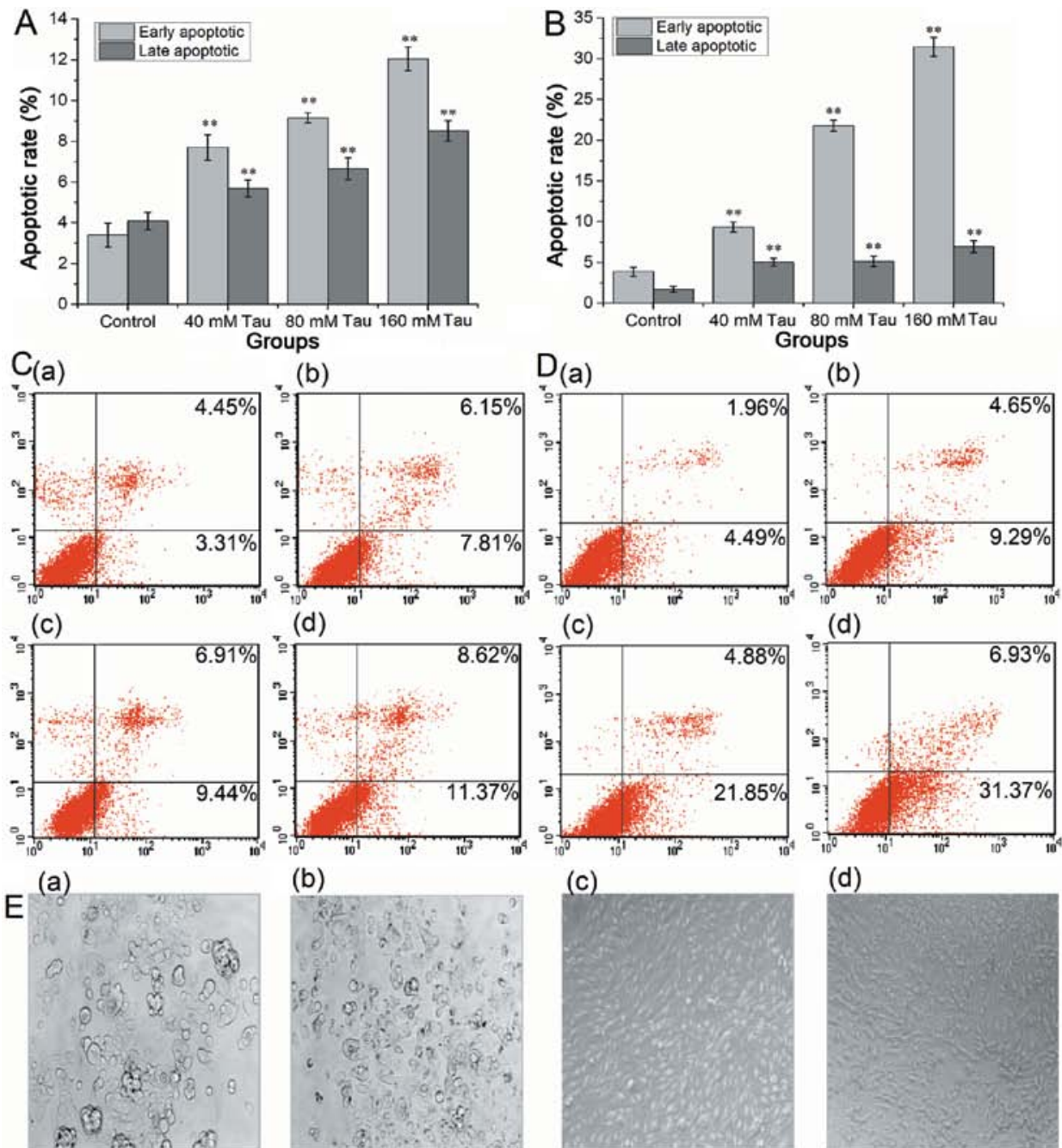

(b)

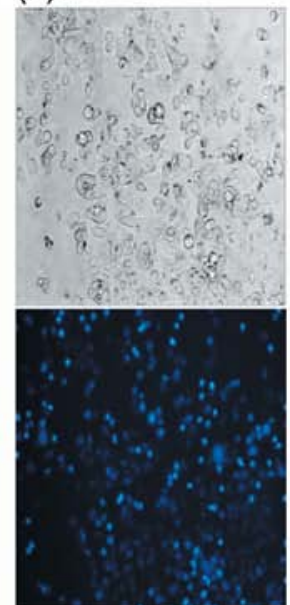

(c)
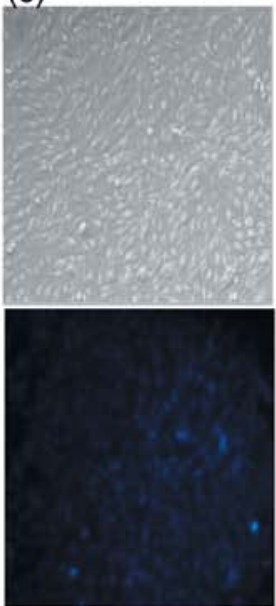

(d)

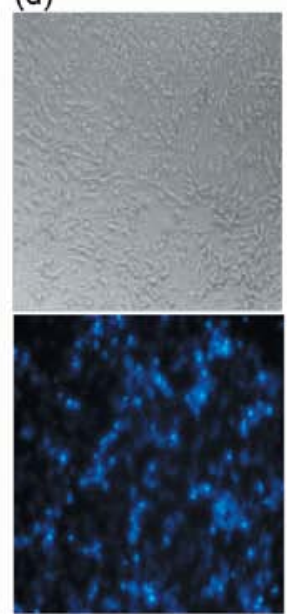

Figure 2. Tau induces apoptosis in MCF-7 and MDA-MB-231 cells. MCF-7 and MDA-MB-231 cells were treated with Tau (40, 80 and $160 \mathrm{mM})$ for 48 h, and apoptotic cells were analyzed by flow cytometry. The statistical analysis of the apoptosis rate of (A) MCF-7 and (B) MDA-MB-231 cells. The apoptotic rates of different groups of (C) MCF-7 and (D) MDA-MB-231 cells. (a) Control group, and (b) 40, (c) 80 and (d) $160 \mathrm{mM}$ Tau treatment. The percentage of apoptotic cells was calculated with WinMDI software. The early and late apoptosis rate from triplicate experiments were collected on a histogram, which included statistics $(\mathrm{n}=3) .{ }^{* *} \mathrm{P}<0.01$ compared to the control group. (E) The changes of morphology in human breast cancer cells by Hoechst33342 (magnification, $200 \mathrm{x}$ ). (a) Control and (b) $80 \mathrm{mM}$ Tau treatment of the MCF-7 cells. (c) Control and (b) $80 \mathrm{mM}$ Tau treatment of the MDA-MB-231 cells. FACS, fluorescein isothiocyanate.
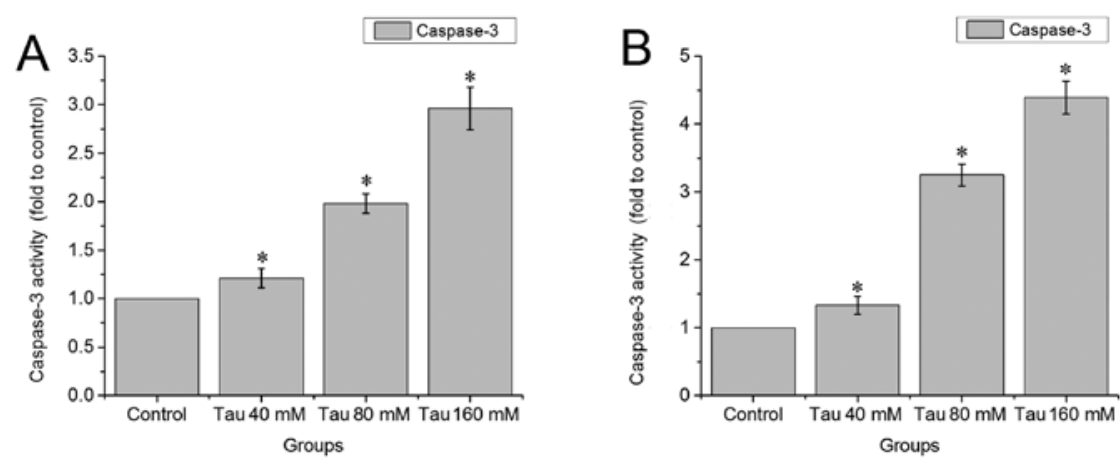

Figure 3. Effect of Tau on caspase-3 activity in MCF-7 and MDA-MB-231 cells. (A) MCF-7 and (B) MDA-MB-231 cells. Date are expressed as the means \pm SD, $\mathrm{n}=3$. ${ }^{*} \mathrm{P}<0.05$ compared to the control group. 
$\mathrm{A}(\mathrm{a}) \quad \begin{array}{lllll}1 & 2 & 3 & 4 & 5\end{array}$
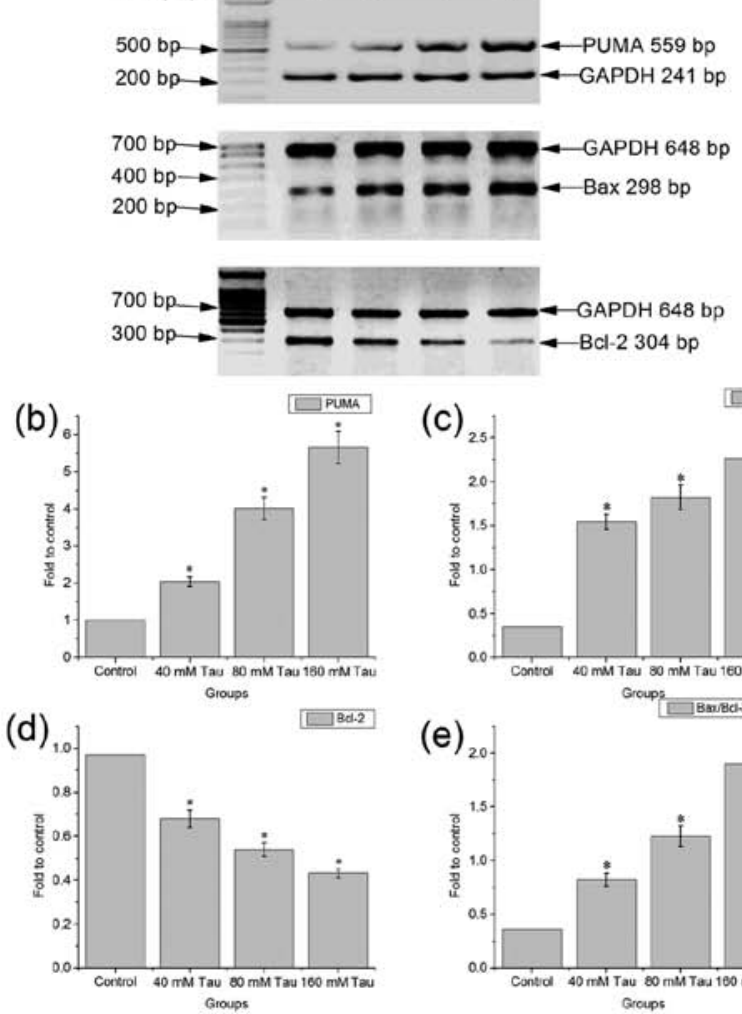

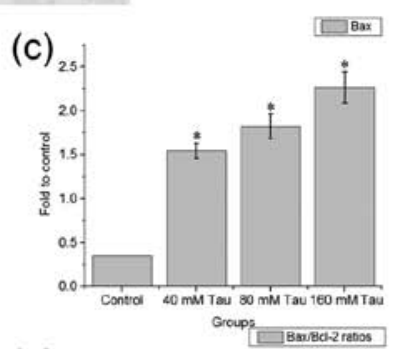

$(e)_{20}$

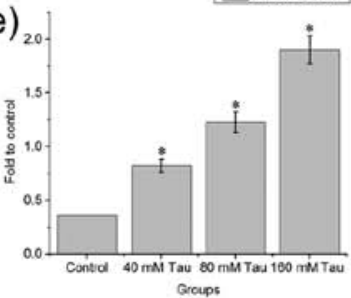

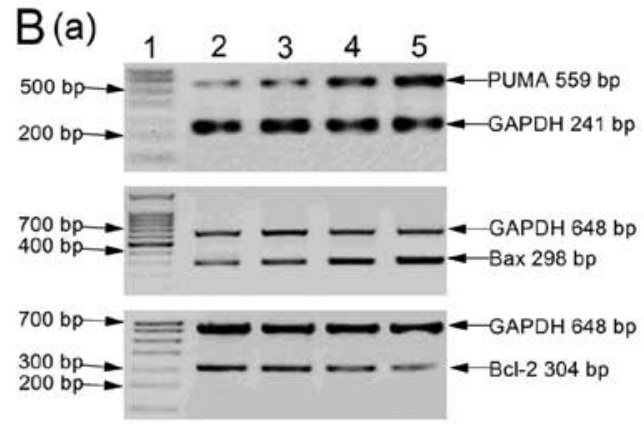
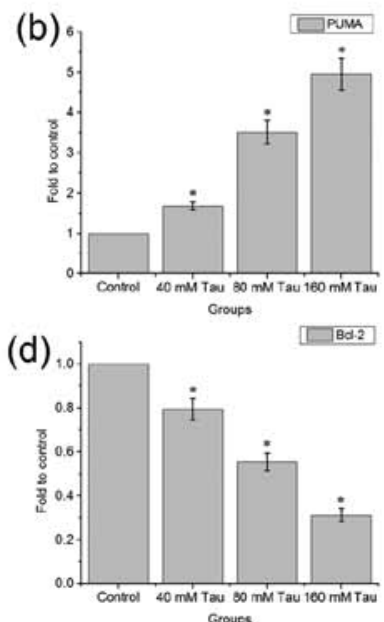

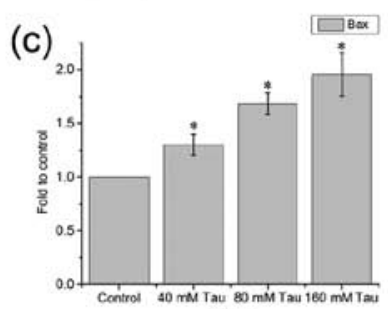

(e)

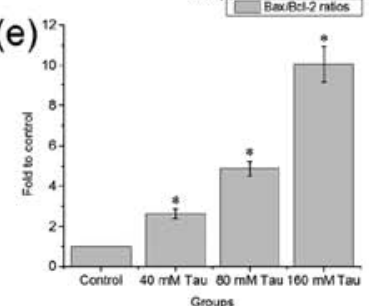

Figure 4. RT-PCR analysis of PUMA, Bax, and Bcl-2 in human breast cancer cells. (A) mRNA expression of MCF-7 and (B) MDA-MB-231 cells. (a) RT-PCR analysis of PUMA, Bax and Bcl-2 treated by Tau. (b-d) Relative levels of PUMA, Bax and Bcl-2 mRNA expression, normalized to value obtained for GAPDH mRNA expression. (e) The ratios of Bax/Bcl-2. Lane 1, DNA marke; lane 2, control group; lane 3, $40 \mathrm{mM}$; lane 4, $80 \mathrm{mM}$; lane 5, $160 \mathrm{mM}$ Tau group. Values are expressed as the means $\pm \mathrm{SD}, \mathrm{n}=3$. ${ }^{*} \mathrm{P}<0.05$ compared to the control group. RT-PCR, reverse transcription-polymerase chain reaction; bp, base pair.

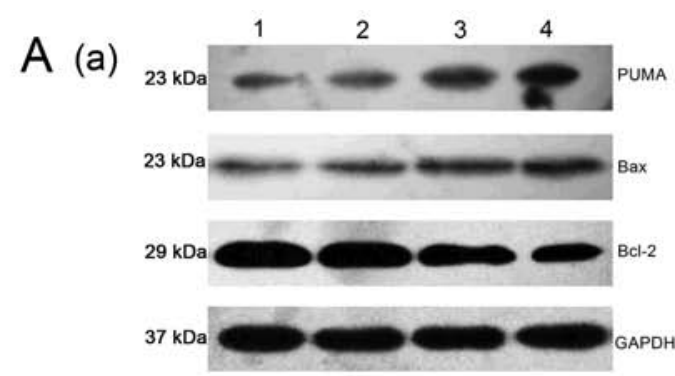

(b)

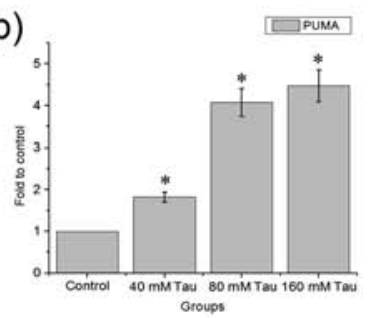

(d)

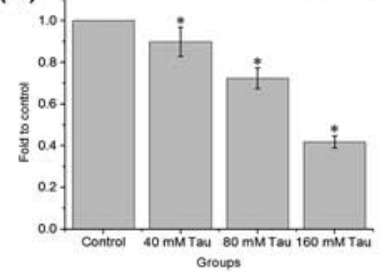

$(c)$

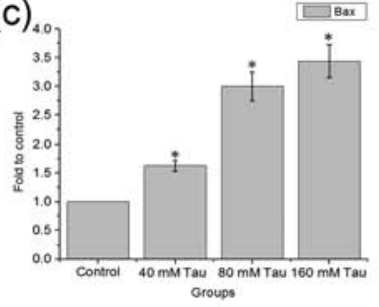

$(\mathrm{e})$

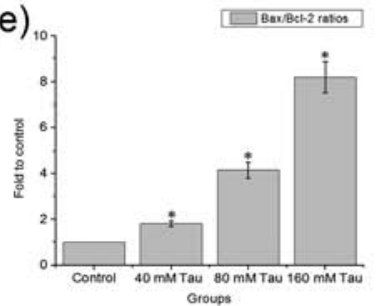

B (a)

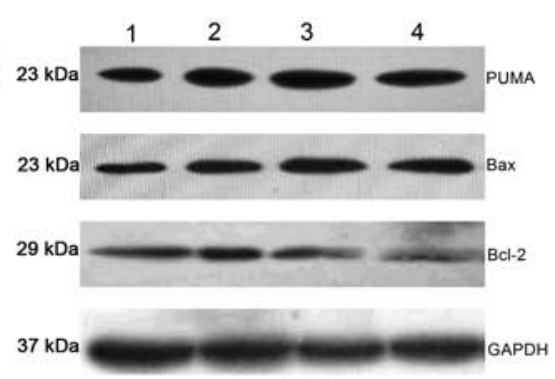

(b)

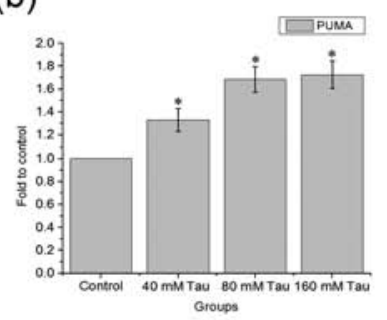

(c)
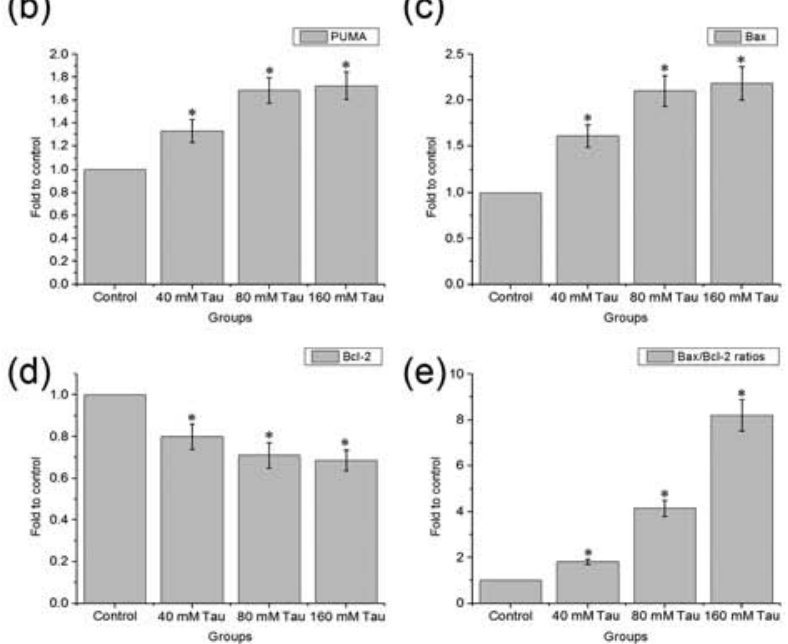

Figure 5. Western blot analysis of PUMA, Bax, and Bcl-2 in human breast cancer cells. (A) Proteins expression of MCF-7 and (B) MDA-MB-231 cells. (a) Western blot analysis of PUMA, Bax and Bcl-2 treated by Tau. (b-d) Relative levels of PUMA, Bax and Bcl-2 proteins expression, normalized to GAPDH protein expression. (e) The ratios of Bax/Bcl-2. Lane 1, control group; lane 2, $40 \mathrm{mM}$; lane 3, $80 \mathrm{mM}$; lane 4, $160 \mathrm{mM}$ Tau group. Values are expressed as the means $\pm \mathrm{SD}, \mathrm{n}=3$. ${ }^{*} \mathrm{P}<0.05$ compared to the control group. 

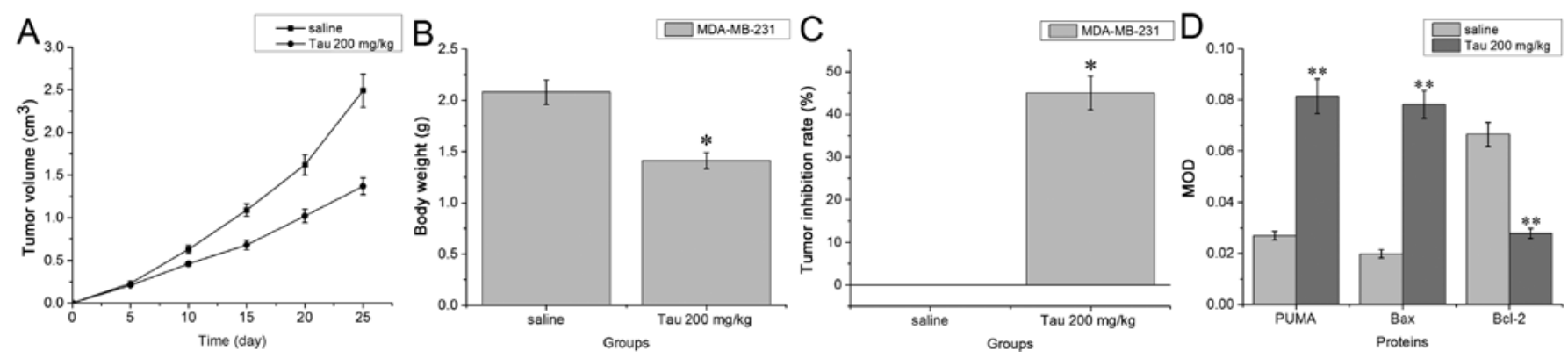

Figure 6. Effect of Tau on the growth of human breast cancer cell-nude mice xenografts. (A) The growth curve of the tumor in nude mice bearing MDA-MB-231 cells. (B) The average tumor weight of MDA-MB-231 cell xenografts after 25 days of treatment. (C) The tumor inhibition rate after 25 days of treatment. Values are expressed as the means $\pm \mathrm{SD}, \mathrm{n}=5$. ${ }^{*} \mathrm{P}<0.05$ compared to the saline group. (D) Statistical analysis of PUMA, Bax and Bcl-2 expression by the mean optical density $(\mathrm{MOD}) . \mathrm{MOD}=$ integral optical density/total area of the image. Values are expressed as the means $\pm \mathrm{SD}, \mathrm{n}=50 .{ }^{* *} \mathrm{P}<0.01$ compared to the saline group.
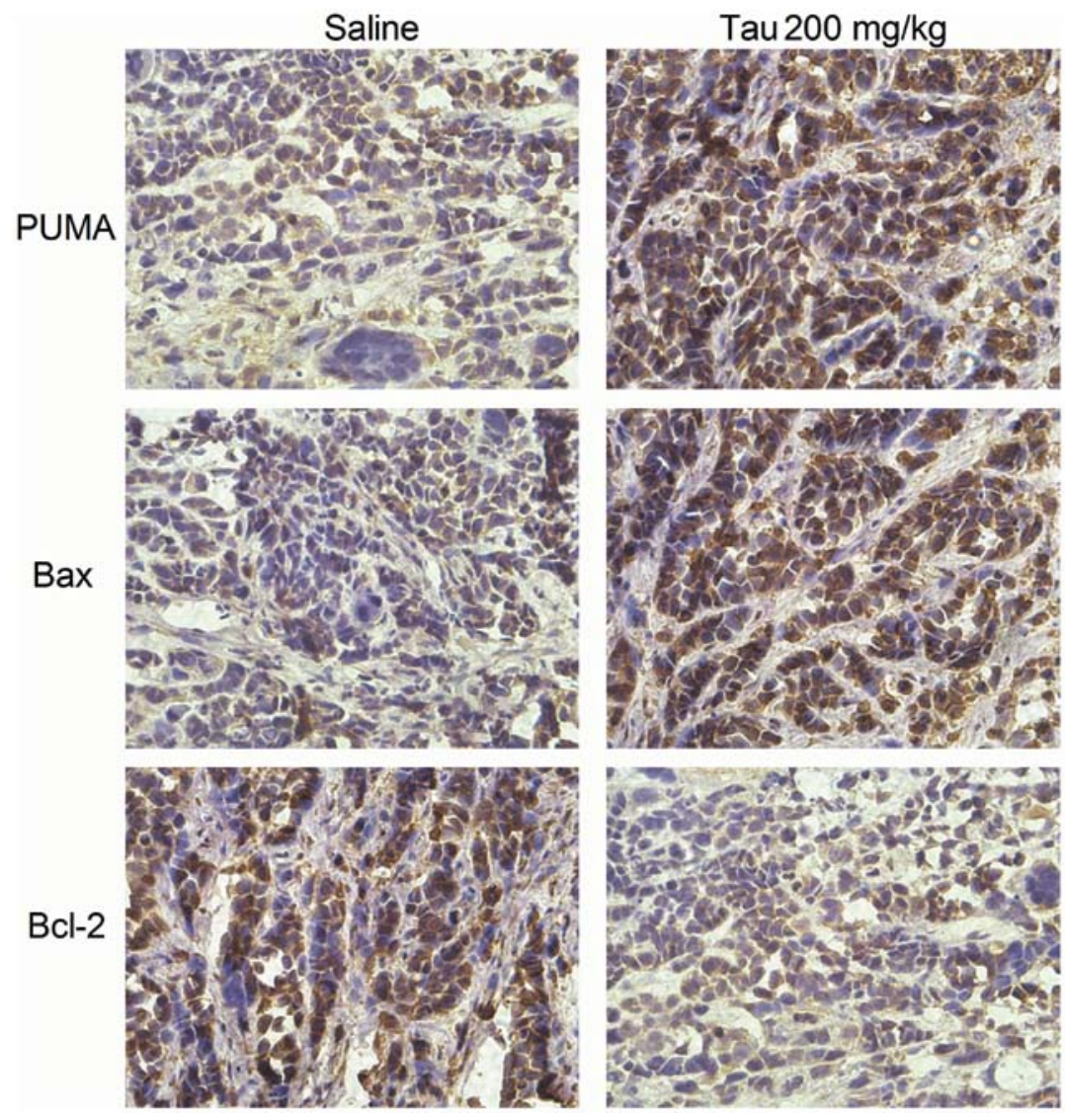

Figure 7. Immunohistochemistry of PUMA, Bax and Bcl-2 expression in MDA-MB-231 xenografts (magnification, $\mathrm{x} 400$ ).

\section{Discussion}

Currently, the changes in the level of Tau in the human body can predict the development and malignancy of the tumor. The assessment level of Tau in the sera of patients with a high risk for breast cancer have been suggested to be of great value in the early diagnosis of malignant changes in the breast (22). The measurement of serum Tau in females with irregular uterine bleeding could help the early detection of malignant transformation of the endometrial wall (23). The significant elevation in Tau concentration was observed in the urine of bladder cancer patients (24). Currently, it is thought that the anti-cancer effect of Tau is mainly through four molecular mechanisms: First, Tau has an efficacy-enhancing and toxicity-reducing effect on chemotherapy drugs (25-28). Second, Tau inhibits cancer growth by improving the antioxidant capacity in vivo (29). Third, Tau plays a role in immune rejection of cancer by enhancing the immune system in vivo (30). Fourth, Tau inhibits the growth of cancer by inducing apoptosis in cancer cells (31).

In the present study, the anti-cancer effect of Tau was investigated with regards to apoptosis. The results proved that Tau significantly inhibited the cell proliferation and induced cell apoptosis in human breast cancer cells, and Tau inhibited the tumor growth in MDA-MB-231 cells-nude mice xenografts. Notably, the present results demonstrated that Tau did not reduce the activity of human embryonic kidney $293 \mathrm{~T}$ cells. This observation has significant implications as an ideal 
chemopreventive agent should be able to eliminate cancer cells while exerting nearly no toxic effect on normal cells.

PUMA is important in drug-induced apoptosis of cancer cells. A variety of chemotherapy drugs can activate the PUMA expression, further inducing apoptosis. Cisplatin-induced PUMA expression has been reported as time- and dose-dependent and its effect on inducing apoptosis in rectal cancer cells is suppressed following antisense lipofection (32). Detection of the expression of multiple genes in locally advanced breast cancer tissue prior and subsequent to doxorubicin chemotherapy showed that the changes mostly occurred in PUMA gene expression (33). Thus far, a variety of traditional Chinese medicines have been shown to have anti-tumor effect, and their pro-apoptotic role is closely associated with the PUMA expression. Previous studies demonstrated that the PUMA expression was increased during the allicin induction of apoptosis in colorectal cancer LoVo cells. The same results were observed in the study by Wang et al (34), which treated the LoVo cells with green tea polyphenols. In the LNCaP prostate cancer cells treated with wogonin, the PUMA protein expression was increased and cytochrome $\mathrm{C}$ was released from mitochondria, leading to the activation of caspase-cascade reaction (35). The antitumor activity of coumarin-chalcone hybrid is mediated through the intrinsic apoptotic pathway by inducing PUMA and altering the Bax/Bcl-2 ratio (36).

The molecular mechanisms of Tau-induced apoptosis in cancer cells were further explored in the present study. The results suggested that Tau possibly upregulated PUMA expression, further upregulated Bax expression, and downregulated Bcl-2 expression, eventually leading to the increased activity of caspase-3 for mediation of apoptosis in breast cancer cells. These findings provide a novel molecular basis for the antitumor effect of Tau.

Numerous studies have reported that the process of PUMA-induced apoptosis was associated with the p53 status. Transfection with wild-type 553 to SW48, SW480 and HCT116 cells found that the expression of PUMA was increased. However, transfection with mutant-type $p 53$, resulted with no change in the expression (37). Another study reported that the DNA-binding domain of p53 directly interacted with the $\mathrm{BH} 3$ domains of PUMA (38). In human colon cancer cells with wild-type p53, crizotinib induces rapid induction of PUMA accompanied by p53 stabilization. The induction of PUMA is mediated largely by p53, and deficiency of p53 blocks crizotinib-induced apoptosis (39). However, an increasing number of studies have found that PUMA can be out of the p53-dependent status. Without considering the state of p53, PUMA adenovirus increases the sensitivity of esophageal cancer cells to anticancer drugs (40). Bryostatin 5 significantly increased the expression of PUMA but slightly increased p53 expression in acute monocytic leukemia cells (41). Estrogen receptor $\beta$ induces apoptosis through PUMA, independent of p53 in prostate cancer cells (42).

In the present study, Tau not only induced apoptosis, but also increased the PUMA expression in MCF-7 and MDA-MB-231 cells. This result indicates that the effect of Tau on inducing the PUMA expression is not associated with the status of p53. Notably, Tau had more significant effects on inhibition of the proliferation and inducing the apoptosis of $\mathrm{p} 53^{-/}$compared to $\mathrm{p} 53^{+/+}$cancer cells. The detailed molecular mechanism remains to be elucidated.
In conclusion, Tau inhibited the proliferation of human breast cancer cells by inducing apoptosis. Regarding the molecular mechanisms, Tau induced PUMA expression, which further up- and downregulated the expression of pro-apoptotic protein Bax and anti-apoptotic protein $\mathrm{Bcl}-2$, giving rise to increased activation of caspase-3. In addition, Tau has a clear effect on inhibiting the $\mathrm{p} 53^{-/-}$cancer cells, suggesting that Tau can upregulate the PUMA gene expression in the MCF-7 and MDA-MB-231 cells, and that the upregulation of PUMA gene expression by Tau is independent of the $p 53$ status.

\section{Acknowledgements}

The present study was supported by the grant from the National Natural Science Foundation of China (no. 81360032).

\section{References}

1. Wogan GN, Hecht SS, Felton JS, Conney AH and Loeb LA: Environmental and chemical carcinogenesis. Semin Cancer Biol 14: 473-486, 2004.

2. Megaraj V, Lida T, Jungsuwadee P, Hofmann AF and Vore M: Hepatobiliary disposition of 3alpha, 6alpha, 7alpha, 12alpha-tetrahydroxy-cholanoyl taurine: a substrate for multiple canalicular transporters. Drug Metab Dispos 38: 1723-1730, 2010.

3. Yin Y, Wen K, Wu Y, Kang Y and Lou J: Inhibition of sodium current by taurine magnesium coordination compound prevents cesium chloride-induced arrhythmias. Biol Trace Elem Res 146: 192-198, 2012.

4. EI Zahraa Z, EI Ashry F, Mahmoud MF, EI Maraghy NN and Ahmed AF: Effect of cordyceps sinensis and taurine either alone or in combination on streptozotocin induced diabetes. Food Chem Toxicol 50: 1159-1165, 2012.

5. Das J, Vasan V and Sil PC: Taurine exerts hypoglycemic effect in alloxan-induced diabetic rats, improves insulin-mediated glucose transport signaling pathway in heart and ameliorates cardiac oxidative stress and apoptosis. Toxicol Appl Pharmacol 258: 296-308, 2012.

6. Son HY, Kim H and H Kwon Y: Taurine prevents oxidative damage of high glucose-induced cataractogenesis in isolated rat lenses. J Nutr Sci Vitaminol (Tokyo) 53: 324-330, 2007.

7. Song XD, Chen CZ, Dong B, Shi YY, Zhang W, Yan LS and Luo GA: Study on the intervening mechanism of taurine on streptozotocin-induced diabetic cataracts. Zhanghua Yan Ke Za Zhi 39: 605-609, 2003 (In Chinese).

8. Mates JM, Segura JA, Alonso FJ and Marguez J: Oxidative stress in apoptosis and cancer: an update. Arch Toxicol 86: 1649-1665, 2012.

9. Mates JM, Segura JA, Alonso FJ and Marguez J: Sulphur-containing non enzymatic antioxidants: therapeutic tools against cancer. Front Biosci (Schol Ed) 4: 722-748, 2012.

10. Neary PM, Hallihan P, Wang JH, Pfirrmann RW, BouchierHayes DJ and Redmond HP: The evolving role of taurolidine in cancer therapy. Ann Surg Oncol 17: 1135-1143, 2010.

11. Letai A: Puma strikes BAX. J Cell Biol 185: 189-190, 2009.

12. Yee KS and Vousden KH: Contribution of membrane localization to the apoptotic activity of puma. Apoptosis 13: 87-95, 2008.

13. Jeffers JR, Parganas E, Lee Y, Yang C, Wang J, Brennan J, MacLean KH, Han J, Chittenden T, Ihle JN, McKinnon PJ, Cleveland JL and Zambetti GP: PUMA is an essential mediator of p53-dependent and -independent apoptotic pathways. Cancer Cell 4: 321-328, 2003.

14. Yu J and Zhang L: The transcriptional targets of p53 in apoptosis control. Biochem Biophys Res Commun 331: 851-858, 2005.

15. Han J, Flemington C, Houghton AB, Gu Z, Zambetti GP, Lutz RJ, Zhu L and Chittenden T: Expression of bbc3, a pro-apoptotic BH3-only gene, is regulated by diverse cell death and survival signals. Proc Natl Acad Sci USA 98: 11318-11323, 2001.

16. Hershko $\mathrm{T}$ and Ginsberg D: Up-regulation of Bcl-2 homology 3(BH3)-only proteins by E2F1 mediates apoptosis. J Biol Chem 279: 8627-8634, 2004. 
17. Spender LC, Carter MJ, O'Brien DI, Clark LJ, Yu J, Michalak EM, Happo L, Cragg MS and Inman GJ: Transforming growth factor-beta directly induces p53-up-regulated modulator of apoptosis (PUMA) during the rapid induction of apoptosis in myc-driven B-cell lymphomas. J Biol Chem 288: 5198-5209, 2013.

18. Lu H, Hou G, Zhang Y, Dai Y and Zhao H: c-jun transactivates puma gene expression to promote osteoarthritis. Mol Med Rep 9: $1606-1612,2014$

19. Yu J and Zhang L: PUMA, a potent killer with or without p53. Oncogene 27: S71-S83, 2008.

20. Tu S, Liu ZQ, Fu JJ, Zhu WF, Luo DY and Wan FS: Inhibitory effect of p53 upregulated modulator of apoptosis targeting siRNA on hypoxia/reoxygenation-induced cardiomyocyte apoptosis in rats. Cardiology 122: 93-100, 2012.

21. Qiu W, Carson-Walter EB, Liu H, Epperly M, Greenberger JS Zambetti GP, Zhang L and Yu J: PUMA regulates intestinal progenitor cell radiosensitivity and gastrointestinal syndrome. Cell Stem Cell 2: 576-583, 2008.

22. El Agouza IM, Eissa SS, El Houseini MM, El-Nashar DE and Abd El Hameed OM: Taurine: a novel tumor marker for enhanced detection of breast cancer among female patients. Angiogenesis 14: 321-330, 2011.

23. El Agouza IM and Nashar DE: Serum taurine as a marker of endometrial cancer. The Open Women's Health Journal 5: 1-6, 2011

24. Srivastava S, Roy R, Singh S, Kumar P, Dalela D, Sankhwar SN, Goel A and Sonkar AA: Taurine-a possible fingerprint biomarker in non-muscle invasive bladder cancer: a pilot study by $1 \mathrm{H} \mathrm{NMR}$ spectroscopy. Cancer Biomark 6: 11-20, 2010.

25. Yu JS and Kim AK: Effect of combination of taurine and azelaic acid on antimelanogenesis in murine melanoma cells. J Biomed Sci 17 Suppl 1: S45, 2010.

26. Ryu CS, Kwak HC, Lee KS, Kang KW, Oh SJ, Lee KH, Kim HM, Ma JY and Kim SK: Sulfur amino acid metabolism in doxorubicin-resistant breast cancer cells. Toxicol Appl Pharmacol 255: 94-102, 2011.

27. El-Houseini ME, Refaei MO, Amin AI and Abol-Ftouh MA Potential role of curcumin and taurine combination therapy on human myeloid leukemic cells propagated in vitro. Leuk Lymphoma 54: 2281-2287, 2013.

28. Kim T and Kim AK: Taurine enhances anticancer activity of cisplatin in human cervical cancer cells. Adv Exp Med Biol 776: 189-198, 2013.

29. Zhang X, Du W, Shen FL and Wang J: Research on effects of taurine on the transplanted tumor of mice. Wei Sheng Jan Jiu 26 321-324, 1997 (In Chinese)

30. Maher SG, Condron CE, Bouchier-Hayes DJ and Toomey DM: Taurine attenuates CD3/interleukin-2-induced T cell apoptosis in an in vitro model of activation-induced cell death (AICD). Clin Exp Immunol 139: 279-286, 2005
31. Zhang X, Tu S, Wang Y, Xu B and Wan F: Mechanism of taurine-induced apoptosis in human colon cancer cells. Acta Bioch Bioph Sin (Shanghai) 46: 261-272, 2014.

32. Wang H, Qian H, Yu J, Zhang X, Zhang L, Fu M, Liang X, Zhan Q and Lin C: Administration of PUMA adenovirus increases the sensitivity of esophageal cancer cells to anticancer drugs. Cancer Biol Ther 5: 380-385, 2006.

33. Middelburg R, de Haas RR, Dekker H, Kerkhoven RM, Pohlmann PR, Fuentes-Alburo A, Mohar A, Pinedo HM and Lankelma J: Induction of p53 up-regulated modulator of apoptosis messenger RNA by chemotherapeutic treatment of locally advanced breast cancer. Clin Cancer Res 11: 1863-1869, 2005.

34. Wang X, Wang R, Hao MW, Dong K, Wei SH, Lin F, Ren JH and Zhang HZ: The BH3-only protein PUMA is involved in green tea polyphenol-induced apoptosis in colorectal cancer cell lines. Cancer Biol Ther 7: 902-908, 2008.

35. Lee DH, Kim C, Zhang L and Lee YJ: Role of p53, PUMA, and BAX in wogonin-induced apoptosis in human cancer cells. Biochem Pharmacol 75: 2020-2033, 2008.

36. Singh N, Sarkar J, Sashidhara KV, Ali S and Sinha S: Anti-tumour activity of a novel coumarin-chalcone hybrid is mediated through intrinsic apoptotic pathway by inducing PUMA and altering Bax/Bcl-2 ratio. Apoptosis 19: 1017-1028, 2014.

37. Yu J, Zhang L, Hwang PM, Kinzler KW and Vogelstein B: PUMA induces the rapid apoptosis of colorectal cancer cells. Mol Cell 7: 673-682, 2001.

38. Park SY, Jeong MS and Jang SB: In vitro binding properties of tumor suppressor p53 with PUMA and NOXA. Biochem Biophys Res Commun 420: 350-356, 2012.

39. Zheng X, He K, Zhang L and Yu L: Crizotinib induces PUMA-dependent apoptosis in colon cancer cells. Mol Cancer Ther 12: 777-786, 2013

40. Wang X, Li M, Wang J, Yeung CM, Zhang h, Kung HF, Jiang B and Lin MC: The BH3-only protein, PUMA, is involved in oxaliplatin-induced apoptosis in colon cancer cells. Biochem Pharmacol 71: 1540-1550, 2006.

41. Wang Y, Zhang J, Wang Q, Zhang T, Yang Y, Yi Y, Gao G, Dong $\mathrm{H}$, Zhu H, Li Y, Lin H, Tang $\mathrm{H}$ and Chen X: Bryostatin 5 induces apoptosis in acute monocytic leukemia cells by activating PUMA and caspases. Eur J Pharmacol 718: 340-349, 2014.

42. Dey P, Ström A and Gustafsson JA: Estrogen receptor $\beta$ upregulates FOXO3a and causes induction of apoptosis through PUMA in prostate cancer. Oncogene 33: 4213-4225, 2014. 